\title{
An interval type-2 fuzzy TOPSIS using the extended vertex method for MAGDM
}

\author{
Iman Mohamad Sharaf ${ }^{1}$
}

Received: 21 October 2019 / Accepted: 28 November 2019 / Published online: 14 December 2019

(c) Springer Nature Switzerland AG 2019

\begin{abstract}
In this article, the technique of order preference by similarity to an ideal solution (TOPSIS) is modified to handle interval type-2 fuzzy sets (IT2FSs) using the extended vertex method for distance measure. While the existing TOPSIS techniques for IT2FSs depend on the defuzzification of the average decision matrix or the average weighted decision matrix from the very beginning, the proposed method maintains fuzziness in the preference technique up to the hilt to avoid any information distortion which might lead to false ranking. First, the vertex method for distance measure is extended to encompass IT2FSs. The extended vertex method is an efficient simple formula that requires few computations in contrast to other distance measures based on embedding type 1 fuzzy sets or a-cuts that need special algorithms and can be restrictive in applications that require high computations. Second, the fuzzy positive and negative ideal solutions are defined. Then, the relative degree of closeness to the ideal solutions is computed for each alternative using the extended vertex method. As the relative degree of closeness of an alternative increases, its preference increases. Therefore, the preference technique avoids the flaws of the existing techniques and the computations are reduced. Two illustrative examples are given and the results are compared with the results of the existing TOPSIS methods. In light of the results and comparisons, the role of the defined ideal solutions in ranking is clarified.
\end{abstract}

Keywords Fuzzy multi-attributes group decision making · TOPSIS · Distance measure $\cdot$ Interval type-2 fuzzy sets

Mathematics Subject Classification 90B50

\section{Introduction}

In modern decision theory, multiple attributes group decision-making (MAGDM) problems play a vital role. In these problems, the best choice is chosen from a set of alternatives counting on experts' assessments of the alternatives according to their multiple attributes [5]. In many cases, the human evaluations and preferences are often ambiguous and vague. In other cases, some of the evaluation attributes are subjective and qualitative in nature that they cannot be expressed using exact numerical values [17].
The concept of fuzzy sets was introduced to handle vagueness, uncertainty, and imprecision in decisionmaking problems. Type- 1 fuzzy sets (T1FSs) are the early proposed sets. They are characterized by a crisp membership function in the interval $[0,1]$. In many situations it is hard to estimate the exact membership function of fuzzy sets. As a result, T1FSs decrease the flexibility and precision of decision-making in an uncertain environment [12]. Recently, MAGDM methods use more elaborate fuzzy sets, e.g. IT2FSs [4, 22]; and intuitionistic fuzzy sets [7, 11, 16, $27,30]$.

Iman Mohamad Sharaf, iman_sharaf@hotmail.com | ${ }^{1}$ Department of Basic Sciences, Higher Technological Institute, Ahmad Hamdy Street, First District, PO Box 228, Tenth of Ramadan City, Egypt. 
Type-2 fuzzy sets (T2FSs) are an extension of T1FSs. Zadeh [31] proposed T2FSs to express linguistic terms more efficiently than T1FSs. The membership degree of each element in a T2FS is represented by another fuzzy set defined over the interval $[0,1]$. Therefore, the membership function of T2FSs is three dimensional. That provides more degrees of freedom in representing uncertainties in recent models [17]. T2FSs have proven to surpass T1FSs in many applications due to their ability to model uncertainties with greater accuracy [20]. However, T2FSs require complicated and massive tiresome operations [17]. This led to the introduction of fuzzy sets that requires simpler and easier computations than T2FSs.

Sambuc [24] introduced interval-valued fuzzy sets (IVFSs) in which the membership values are expressed by intervals. The exact membership degree lies within the considered interval [28]. Consequently, an IVFS is defined by an upper T1FS membership function and a lower T1FS membership function. Later, Liang and Mendel [19] introduced IT2FSs as a particular case of T2FSs whose secondary membership degree is equal to one. IVFSs can be considered as a special case of IT2FSs in which the membership values are equal in both the upper and lower fuzzy numbers. Although IVFSs and IT2FSs might appear to be similar, they are not totally equivalent. The information capacity of these fuzzy sets is not equal [21]. IT2FSs can represent concepts that cannot be represented by IVFSs. Actually, IT2FSs can be considered as a generalization of IVFSs [28]. For the points of similarity and difference between the two sets, the reader is referred to Niewiadomski [21] and Sola [28].

The technique of order preference by similarity to an ideal solution (TOPSIS) is one of the well-known methods to solve MAGDM problems. TOPSIS is preferred due to its simplicity, intuitiveness and limited computations [15]. It proved to be a useful practical tool for ranking and selecting among alternatives [23]. A TOPSIS solution is an alternative which is the closest to the positive ideal solution (PIS) and the farthest from the negative ideal solution (NIS). First, the weighted ratings are defuzzified into crisp values. Second, their distance from both the positive and negative ideal solutions is calculated. Then, a closeness coefficient is defined to determine the ranking order of the alternatives. Chu and Lin [6] proposed the conversion of the weighted normalized decision matrix to crisp values by defuzzification to change a fuzzy MAGDM problem into a crisp one.

Several modifications have been introduced to fuzzy TOPSIS. These modifications are either in the defuzzification technique or in the preference technique. Defuzzification is simple and easy. Nevertheless, defuzzification loses uncertainty of messages. On the other hand, a fuzzy pair-wise comparison is complex and difficult. However, it preserves fuzziness in messages [15]. Ashtiani et al. [1] proposed a triangular interval-valued fuzzy TOPSIS (IVFTOPSIS) to solve MAGDM problems. Chen and Lee [5] presented an interval type-2 fuzzy TOPSIS (IT2F-TOPSIS) for fuzzy MAGDM problems. The method relies on the early defuzzification of the average weighted decision matrix. This leads to two drawbacks, it gives an incorrect preferred order of the alternatives in some situations, and the preferred order of the alternatives will change if additional alternatives are added [3]. To overcome these drawbacks, Chen and Hong [3] introduced a new ranking technique for IT2FSs and utilized it in TOPSIS. Still, early defuzzfication is carried out for the attributes' fuzzy weights and the average fuzzy decision matrix. Rashid et al. [23] extended TOPSIS for generalized interval-valued trapezoidal fuzzy numbers. Yet, the used heuristic expression to calculate the difference between interval-valued trapezoidal fuzzy numbers was not justified [8]. Therefore, Dymova et al. [8] introduced an interval type-2 fuzzy extension of the TOPSIS method using a-cuts to avoid the limitations and drawbacks of the existing methods. Ilieva [15] used the graded mean integration to defuzzify IT2FSs into two crisp values and then working with their average value. Kumar and Garg [18] proposed a TOPSIS method for interval-valued intuitionistic fuzzy sets based on set pair analysis. Sharaf [25] proposed an IVF-TOPSIS using similarity measure based on map distance for preference comparison.

In this article, TOPSIS is modified to handle IT2FSs using the extended vertex method for distance measure. The existing TOPSIS methods for IT2FSs rely on the early defuzzification of the average decision matrix or the average weighted decision matrix. The proposed method maintains fuzziness in the preference technique to avoid the disadvantages of defuzzification which may lead to incorrect ranking. First, the vertex method for distance measure is extended to include IT2FSs. The proposed distance measure is simple and requires few computations. Meanwhile, the other distance measures based on embedding type 1 fuzzy sets or a-cuts need special algorithms and can be restrictive in applications that require high computations. The performance of the vertex method is compared versus other distance measures. Second, the fuzzy positive and negative ideal solutions are defined for IT2FSs. Then, the relative degree of closeness to the ideal solutions is computed for each alternative using the proposed distance measure. As the relative degree of closeness of an alternative increases, its preference increases. Therefore, the preference technique depends on a fuzzy basis to avoid the flaws of the existing techniques resulting from the loss of information due to defuzzification and the computations are reduced due to the simple and efficient formula of the extended vertex method for distance measure. 
The article is organized as follows. Different types of fuzzy sets, distance measures, and the classical TOPSIS are presented in Sect. 2. The extended vertex method is introduced in Sect. 3. The proposed TOPSIS method is introduced in Sect. 4. Two illustrative examples are given to demonstrate the approach; and the results are compared with the results of some existing TOPSIS methods in Sect. 5. Discussion is given in Sect. 6 . Finally, the conclusion is given in Sect. 7 .

\section{Preliminaries}

\subsection{Interval type-2 fuzzy numbers}

A type-2 fuzzy set is represented by

$$
\tilde{\mathrm{A}}=\int_{\forall x \in \boldsymbol{X}} \int_{\forall u \in J_{x} \subseteq[0,1]} \mu_{\tilde{A}}(x, u) /(x, u),
$$

where $\mu_{\tilde{A}}(x, u)$ is a type-2 membership function and $\iint$ denotes the union over all admissible $x$ and $u$ [8]. IT2FS is a T2FS with $\mu_{\tilde{A}}(x, u)=1$.

IT2FS are represented using two trapezoidal fuzzy numbers as follows:

$\tilde{A}=\left[\tilde{A}^{L}, \tilde{A}^{U}\right]=\left[\left(a_{1}^{L}, a_{2}^{L}, a_{3}^{L}, a_{4}^{L} ; w_{1}^{L}, w_{2}^{L}\right),\left(a_{1}^{U}, a_{2}^{U}, a_{3}^{U}, a_{4}^{U} ; w_{1}^{U}, w_{2}^{U}\right)\right]$,

where $a_{1}^{L}, a_{2}^{L}, a_{3}^{L}, a_{4}^{L}, a_{1}^{U}, a_{2}^{U}, a_{3}^{U}, a n d a_{4}^{U} \in R$ (the set of real numbers) are the reference points of the IT2FS, and $w_{1}^{L}, w_{2}^{L}, w_{1}^{U}$ and $w_{2}^{U} \in[0,1]$ denote the membership values [17]. Trapezoidal IVFS is a special case of trapezoidal IT2FS when $w_{1}^{L}=w_{2}^{L}$ and $w_{1}^{U}=w_{2}^{U}$.

For the two interval type-2 fuzzy numbers $\tilde{A}=\left[\left(a_{1}^{L}, a_{2}^{L}, a_{3}^{L}, a_{4}^{L} ; w_{1 \tilde{A}^{\prime}}^{L}, w_{2 \tilde{A}}^{L}\right),\left(a_{1}^{U}, a_{2}^{U}, a_{3}^{U}, a_{4}^{U} ; w_{1 \tilde{A}^{\prime}}^{U}, w_{2 \tilde{A}}^{U}\right)\right]$ and $\tilde{B}=\left[\left(b_{1}^{L}, b_{2}^{L}, b_{3}^{L}, b_{4}^{L} ; w_{1 \tilde{B}^{\prime}}^{L}, w_{2 \tilde{B}}^{L}\right),\left(b_{1}^{U}, b_{2}^{U}, b_{3}^{U}, b_{4}^{U} ; w_{1 \tilde{B}^{\prime}}^{U}, w_{2 \tilde{B}}^{U}\right)\right]$, the aggregation operations are defined as follows [5]:

\subsection{Distance measure}

\subsubsection{Concept of distance measures}

Distance measures have been extensively studied due to their applications in multiple areas, e.g. risk analysis, data mining, signal processing and pattern recognition $[26,27]$. A distance measure depicts the difference between two fuzzy sets. Computing the difference between two fuzzy sets as a crisp number is crucial for ranking and preference. Yet as the distance is computed in an inaccurate domain, due to vagueness, a rational problem arises [13].

Definition 2.2.1.1 [13] A real function " $d$ " is called a metric distance, if " $d$ " satisfies the following properties

1. $d(\tilde{A}, \tilde{B}) \geq 0$, for any two IT2FSs $\tilde{A}$ and $\tilde{B}$.

2. $d(\tilde{A}, \tilde{B})=d(\tilde{B}, \tilde{A})$, for any two IT2FSs $\tilde{A}$ and $\tilde{B}$.

3. $d(\tilde{A}, \tilde{B})+d(\tilde{B}, \tilde{C}) \geq d(\tilde{A}, \tilde{C})$, for any three IT2FSs $\tilde{A}, \tilde{B}$ and $\tilde{C}$.

Definition 2.2.1.2 [13] for any three IT2FSs $\tilde{A}, \tilde{B}$ and $\tilde{C}$, we can write $\tilde{A}<\tilde{B}<\tilde{C}$ if and only if $d(\tilde{A}, \tilde{B})<d(\tilde{A}, \tilde{C})$, which means that $\tilde{B}$ is closer to $\tilde{A}$ than $\tilde{C}$.

Most of the distance measures for IT2FSs are generalizations of the distances used in the crisp sets, replacing the characteristic functions by the membership functions, e.g. the normalized Hamming distance, the normalized Euclidean distance and the normalized Hamming distance based on Hausdorff metric. Heidarzade et al. [13] demonstrated that these three distance measures are not appropriate for IT2FSs.

Figueroa-Garcia and Hernandez-Perez [10] proposed a distance measure for triangular IT2FSs, i.e. with triangular lower and upper membership functions, using its decomposition

$\tilde{A} \oplus \tilde{B}=\left[\left(a_{1}^{L}+b_{1}^{L}, a_{2}^{L}+b_{2}^{L}, a_{3}^{L}+b_{3^{\prime}}^{L} a_{4}^{L}+b_{4}^{L} ; \min \left(w_{1 \tilde{A}^{\prime}}^{L} w_{1 \tilde{B}}^{L}\right), \min \left(w_{2 \tilde{A}^{\prime}}^{L} w_{2 \tilde{B}}^{L}\right)\right),\left(a_{1}^{U}+b_{1}^{U}, a_{2}^{U}+b_{2}^{U}, a_{3}^{U}+b_{3}^{U}, a_{4}^{U}+b_{4}^{U} ; \min \left(w_{1 \tilde{A}^{\prime}}^{U} w_{1 \tilde{B}}^{U}\right), \min \left(w_{2 \tilde{A}^{\prime}}^{U}, w_{2 \tilde{B}}^{U}\right)\right)\right]$.

$\tilde{A} \oplus \tilde{B}=\left[\left(a_{1}^{L} b_{1}^{L}, a_{2}^{L} b_{2}^{L}, a_{3}^{L} b_{3}^{L}, a_{4}^{L} b_{4}^{L} ; \min \left(w_{1 A^{\prime}}^{L}, w_{1 B}^{L}\right), \min \left(w_{2 A^{\prime}}^{L}, w_{2 B}^{L}\right)\right),\left(a_{1}^{U} b_{1}^{U}, a_{2}^{U} b_{2}^{U}, a_{3}^{U} b_{3}^{U}, a_{4}^{U} b_{4}^{U} ;\left(w_{1 A^{\prime}}^{U}, w_{1 B}^{U}\right), \min \left(w_{2 A^{\prime}}^{U}, w_{2 B}^{U}\right)\right)\right]$.

For an arbitrary real number $k$

$$
\begin{aligned}
& k . \tilde{A}=\tilde{A} \cdot k
\end{aligned}
$$

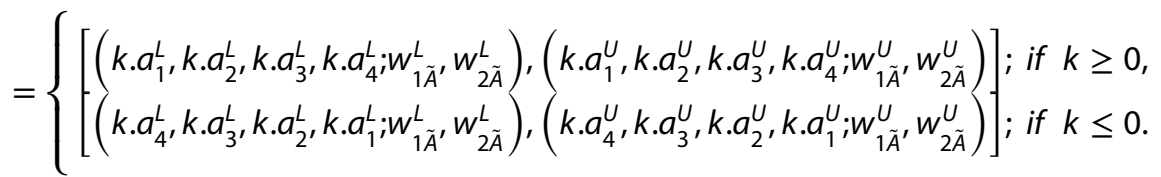


into $a$-cuts. However, the a-cuts based distance can be restrictive in applications that require high computational efforts [9]. For this reason, Figueroa-Garcia et al. [9] proposed centroid based distance measures for triangular IT2FSs. However, centroids are a sort of defuzzification, regardless the formula used for measuring distance based on them.

Heidarzade et al. [13] proposed a distance measure for IT2FSs. The algorithm assumes $n$ embedded type- 1 fuzzy numbers within the surface of the footprint of uncertainty (FOU). By increasing $n$, the surface of FOU is embedded with more type- 1 fuzzy sets. Therefore, the value of $n$ has a direct impact on the difference between the upper and lower membership functions for the IT2FS.

$$
\mathbf{D}=\begin{gathered}
\mathrm{x}_{1} \\
f_{1} \\
f_{2} \\
\vdots \\
f_{m}
\end{gathered}\left[\begin{array}{ccccc}
f_{11} & f_{12} & & & \mathrm{x}_{\mathrm{n}} \\
f_{21} & f_{22} & \cdots & f_{1 n} \\
& \vdots & & \ddots & f_{2 n} \\
f_{m 1} & & f_{m 2} & \cdots & f_{m n}
\end{array}\right],
$$

the TOPSIS method as initially developed by Hwang and Yoon (1981) can be summarized as follows.

Step 1. Form the normalized decision matrix. In this step, the various attribute dimensions are transformed into non-dimensional attributes to allow comparing the attributes.

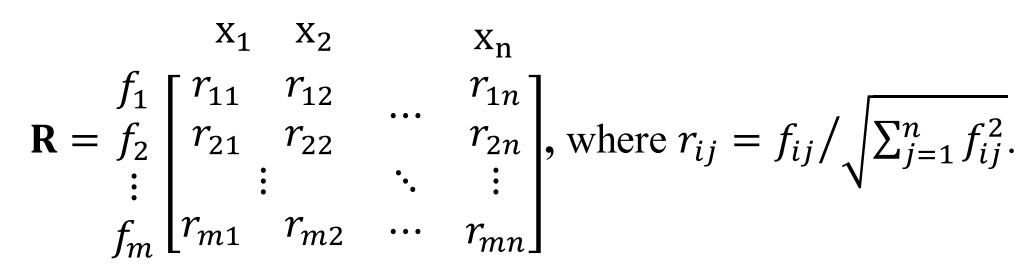

For details on distance measures for IT2FSs, the reader is referred to Zhang et al. [32], Figueroa-Garcia et al. [9] and Heidarzade et al. [13].

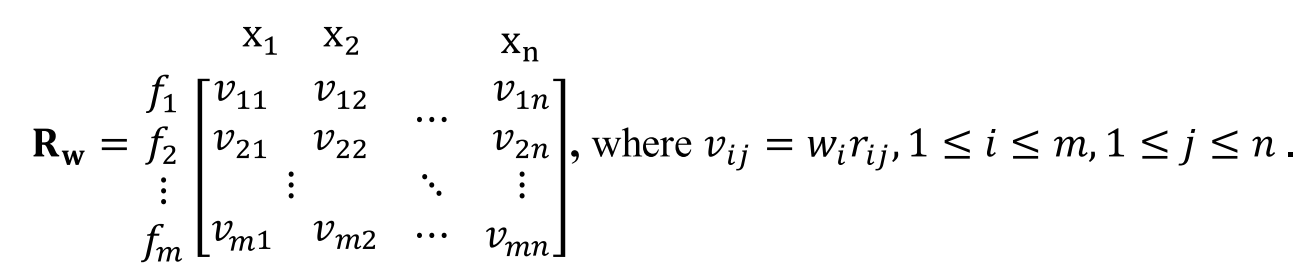

Step 3. Determine the positive and negative ideal solu-

tions.

$$
\begin{aligned}
& \boldsymbol{v}^{+}=\left\{\left(\max _{j} \boldsymbol{v}_{i j} \mid j \in F_{b}\right),\left(\min _{j} \boldsymbol{v}_{i j} \mid j \in F_{c}\right) \mid j=1,2, \ldots n\right\}=\left(v_{1}^{+}, v_{2}^{+}, \ldots v_{m}^{+}\right), \\
& \boldsymbol{v}^{-}=\left\{\left(\min _{j} \boldsymbol{v}_{i j} \mid j \in F_{b}\right),\left(\max _{j} \boldsymbol{v}_{i j} j \in F_{c}\right) \mid j=1,2, \ldots n\right\}=\left(v_{1}^{-}, v_{2}^{-}, \ldots v_{m}^{-}\right),
\end{aligned}
$$

\subsection{The classical TOPSIS}

Given a decision matrix $\mathbf{D}$ that contains $n$ alternatives and $m$ attributes,
Step 2. Form the weighted normalized decision matrix. Since we can't assume that all the criteria are of equal importance, the decision-makers assign a set of weights $w=\left(w_{1}, w_{2}, \ldots, w_{m}\right)$ for the criteria. Each criterion is multiplied by its associated weight where $F_{b}$ are the benefit criteria, and $F_{c}$ are the cost criteria.

Step 4. Calculate the separation measures. 
The Euclidean distance is used to compute the separation measures between each alternative and the ideal solutions.

$$
S_{j}^{+}=\sqrt{\sum_{i=1}^{m}\left(v_{i j}-v^{+}\right)^{2}}, S_{j}^{-}=\sqrt{\sum_{i=1}^{m}\left(v_{i j}-v^{-}\right)^{2}}, j=1,2, \ldots n .
$$

Step 5. Calculate the relative closeness to the ideal solution.

$R_{j}=\frac{S_{j}^{-}}{S_{j}^{+}+S_{j}^{-}}, j=1,2, \ldots n$

Step 6. Rank the preference order.

The alternatives can be ranked to the descending order of the relative closeness.

\section{The extended vertex method}

Chen [2] extended the TOPSIS method for group decision making under fuzzy environment. To find the relative degree of closeness, the distance to both the PIS and the NIS must be calculated. Chen [2] proposed the vertex

$$
\begin{aligned}
d^{2}\left(\tilde{A}^{L}, \tilde{B}^{L}\right)= & \left(a_{1}^{L}-b_{1}^{L}\right)^{2}+\left(a_{2}^{L}-b_{2}^{L}\right)^{2}+\left(w_{1 \tilde{A}}^{L}-w_{1 \tilde{B}}^{L}\right)^{2} \\
& +\left(a_{3}^{L}-b_{3}^{L}\right)^{2}+\left(w_{2 \tilde{A}}^{L}-w_{2 \tilde{B}}^{L}\right)^{2}+\left(a_{4}^{L}-b_{4}^{L}\right)^{2} .
\end{aligned}
$$

The sum of square the distances between the vertices of the upper membership functions, $\left(a_{1}^{U}, 0\right),\left(a_{2}^{U}, w_{1 \tilde{A}}^{L}\right)$ $\left(a_{3}^{U}, w_{2 \tilde{A}}^{L}\right)\left(a_{4}^{U}, 0\right)$ and $\left(b_{1}^{U}, 0\right),\left(b_{2}^{U}, w_{1 \tilde{B}}^{U}\right)\left(b_{3}^{U}, w_{2 \tilde{B}}^{L}\right)\left(b_{4}^{U}, 0\right)$, is given by:

$$
\begin{aligned}
d^{2}\left(\tilde{A}^{U}, \tilde{B}^{U}\right)= & \left(a_{1}^{U}-b_{1}^{U}\right)^{2}+\left(a_{2}^{U}-b_{2}^{U}\right)^{2}+\left(w_{1 \tilde{A}}^{U}-w_{1 \tilde{B}}^{U}\right)^{2} \\
& +\left(a_{3}^{L}-b_{3}^{U}\right)^{2}+\left(w_{2 \tilde{A}}^{U}-w_{2 \tilde{B}}^{U}\right)^{2}+\left(a_{4}^{U}-b_{4}^{U}\right)^{2} .
\end{aligned}
$$

Definition 2.3.2.2 For the two interval type-2 fuzzy numbers

$\tilde{A}=\left[\left(a_{1}^{L}, a_{2}^{L}, a_{3}^{L}, a_{4}^{L} ; w_{1 \tilde{A}^{\prime}}^{L}, w_{2 \tilde{A}}^{L}\right),\left(a_{1}^{U}, a_{2}^{U}, a_{3}^{U}, a_{4}^{U} ; w_{1 \tilde{A}^{\prime}}^{U} w_{2 \tilde{A}}^{U}\right)\right]$

and

$\tilde{B}=\left[\left(b_{1}^{L}, b_{2}^{L}, b_{3}^{L}, b_{4}^{L} ; w_{1 \tilde{B}^{\prime}}^{L}, w_{2 \tilde{B}}^{L}\right),\left(b_{1}^{U}, b_{2}^{U}, b_{3}^{U}, b_{4}^{U} ; w_{1 \tilde{B}^{\prime}}^{U}, w_{2 \tilde{B}}^{U}\right)\right]$

, the distance between them is given by the formula:

$d(\tilde{A}, \tilde{B})=\sqrt{\frac{1}{8}\left[\begin{array}{c}\left(a_{1}^{L}-b_{1}^{L}\right)^{2}+\left(a_{2}^{L}-b_{2}^{L}\right)^{2}+\left(a_{3}^{L}-b_{3}^{L}\right)^{2}+\left(a_{4}^{L}-b_{4}^{L}\right)^{2}+\left(a_{1}^{U}-b_{1}^{U}\right)^{2}+\left(a_{2}^{L}-b_{2}^{U}\right)^{2}+\left(a_{3}^{U}-b_{3}^{U}\right)^{2}+ \\ \left(a_{4}^{U}-b_{4}^{U}\right)^{2}+\left(w_{1 \tilde{A}}^{L}-w_{1 \tilde{B}}^{L}\right)^{2}+\left(w_{2 \tilde{A}}^{L}-w_{2 \tilde{B}}^{L}\right)^{2}+\left(w_{1 \tilde{A}}^{U}-w_{1 \tilde{B}}^{U}\right)^{2}+\left(w_{2 \tilde{A}}^{U}-w_{2 \tilde{B}}^{U}\right)^{2} .\end{array}\right]}$

method to calculate the distance between two triangular type-1 fuzzy numbers. The vertex method is defined as follows.

Definition 2.3.2.1 [2]: For the two triangular type-1 fuzzy numbers $\tilde{m}=\left(\tilde{m}_{1}, \tilde{m}_{2}, \tilde{m}_{3}\right)$ and $\tilde{n}=\left(\tilde{n}_{1}, \tilde{n}_{2}, \tilde{n}_{3}\right)$, the distance between them is defined as

$d(\tilde{m}, \tilde{n})=\sqrt{\frac{1}{3}\left[\left(m_{1}-n_{1}\right)^{2}+\left(m_{2}-n_{2}\right)^{2}+\left(m_{3}-n_{3}\right)^{2}\right]}$.

Generalizing the concept to handle IT2FSs, the extended vertex method is defined as follows.

Consider the fuzzy numbers $\tilde{A}=\left[\left(a_{1}^{L}, a_{2}^{L}, a_{3}^{L}, a_{4}^{L} ; w_{1 \tilde{A}^{\prime}}^{L}, w_{2 \tilde{A}}^{L}\right)\right.$, $\left.\left(a_{1}^{U}, a_{2}^{U}, a_{3}^{U}, a_{4}^{U} ; w_{1 \tilde{A}^{\prime}}^{U}, w_{2 \tilde{A}}^{U}\right)\right]$ and $\tilde{B}=\left[\left(b_{1}^{L}, b_{2}^{L}, b_{3}^{L}, b_{4}^{L} ; w_{1 \tilde{B}^{\prime}}^{L}, w_{2 \tilde{B}}^{L}\right)\right.$, $\left.\left(b_{1}^{U}, b_{2}^{U}, b_{3}^{U}, b_{4}^{U} ; w_{1 \tilde{B}^{\prime}}^{U}, w_{2 \tilde{B}}^{U}\right)\right]$.

The sum of square the distances between the vertices of the lower membership functions, $\left(a_{1}^{L}, 0\right),\left(a_{2}^{L}, w_{1 \tilde{A}}^{L}\right)\left(a_{3}^{L}, w_{2 \tilde{A}}^{L}\right)$ $\left(a_{4}^{L}, 0\right)$ and $\left(b_{1}^{L}, 0\right),\left(b_{2}^{L}, w_{1 \tilde{B}}^{L}\right)\left(b_{3}^{L}, w_{2 \tilde{B}}^{L}\right)\left(b_{4}^{L}, 0\right)$, is given by:
The properties of the metric dist a n c e, $\quad d(\tilde{A}, \tilde{B}) \geq 0, d(\tilde{A}, \tilde{B})=d(\tilde{B}, \tilde{A}), \quad$ a $\mathrm{n} \mathrm{d}$ $d(\tilde{A}, \tilde{B})+d(\tilde{B}, \tilde{C}) \geq d(\tilde{A}, \tilde{C})$, are trivial from the formula.

Proposition 1 If $\tilde{A}$ and $\tilde{B}$ are real numbers, then the distance measure reduces to the Euclidean distance.

Proof If $\tilde{A}$ is a real number, then

$a_{1}^{L}=a_{2}^{L}=a_{3}^{L}=a_{4}^{L}=a_{1}^{U}=a_{2}^{U}=a_{3}^{U}=a_{4}^{U}=a \quad$ a n d $w_{1 \tilde{A}}^{L}=w_{2 \tilde{A}}^{L}=w_{\tilde{1} \tilde{A}}^{U}=w_{2 \tilde{A}}^{U}=1$.

Similarly, if $\tilde{B}$ is a real number, then

$b_{1}^{L}=b_{2}^{L}=b_{3}^{L}=b_{4}^{L}=b_{1}^{U}=b_{2}^{U}=b_{3}^{U}=b_{4}^{U}=b$

and $w_{1 \tilde{B}}^{L}=w_{2 B}^{L}=w_{1 B}^{U}=w_{2 B}^{U}=1$.

Substituting in the extended vertex formula, 


$$
\begin{aligned}
d(\tilde{A}, \tilde{B}) & =\sqrt{\frac{1}{8}\left[\begin{array}{c}
(a-b)^{2}+(a-b)^{2}+(a-b)^{2}+(a-b)^{2}+(a-b)^{2}+(a-b)^{2}+(a-b)^{2} \\
+(a-b)^{2}+(1-1)^{2}+(1-1)^{2}+(1-1)^{2}+(1-1)^{2}
\end{array}\right]} \\
& =\sqrt{\frac{1}{8}\left[8(a-b)^{2}\right]}=|a-b| .
\end{aligned}
$$

Proposition 2 Two IT2FSs $\tilde{A}$ and $\tilde{B}$ are identical if and only ifd $(\tilde{A}, \tilde{B})=0$.

\section{Proof}

1. (i) Let $\tilde{A}=\tilde{B}$, then any two IT2FSs whether normalized or not and the membership values are not necessarily equal. The distances between the first word FOU (None to very little) with the other 31 words FOUs are computed. Since the words are ranked from the smallest to the largest, according to $\mathrm{Wu}$ and Mendel's centroid based ranking, the distances should be in an increasing order. The results are given in Table 1.

$$
a_{1}^{L}=b_{1}^{L}, a_{2}^{L}=b_{2}^{L}, a_{3}^{L}=b_{3}^{L} a_{4}^{L}=b_{4}^{L}, a_{1}^{U}=b_{1}^{U}, a_{2}^{U}=b_{2}^{U}, a_{3}^{U}=b_{3}^{U}, a_{4}^{U}=b_{4}^{U}, w_{1 \tilde{A}}^{L}=w_{1 \tilde{B}^{\prime}}^{L} w_{2 B}^{L}=w_{2 \tilde{A}^{\prime}}^{L}, w_{1 \tilde{A}}^{U}=w_{1 B^{\prime}}^{U} \text {, and } w_{2 \tilde{A}}^{U}=w_{2 B}^{U} .
$$

Substituting in the vertex formula gives $d(\tilde{A}, \tilde{B})=0$.

2. If $d(\tilde{A}, \tilde{B})=0$, then

$$
\sqrt{\frac{1}{8}\left[\begin{array}{c}
\left(a_{1}^{L}-b_{1}^{L}\right)^{2}+\left(a_{2}^{L}-b_{2}^{L}\right)^{2}+\left(a_{3}^{L}-b_{3}^{L}\right)^{2}+\left(a_{4}^{L}-b_{4}^{L}\right)^{2}+\left(a_{1}^{U}-b_{1}^{U}\right)^{2}+\left(a_{2}^{L}-b_{2}^{U}\right)^{2}+\left(a_{3}^{U}-b_{3}^{U}\right)^{2} \\
+\left(a_{4}^{U}-b_{4}^{U}\right)^{2}+\left(w_{1 \tilde{A}}^{L}-w_{1 \tilde{B}}^{L}\right)^{2}+\left(w_{2 \tilde{A}}^{L}-w_{2 \tilde{B}}^{L}\right)^{2}+\left(w_{1 \tilde{A}}^{U}-w_{1 \tilde{B}}^{U}\right)^{2}+\left(w_{2 \tilde{A}}^{U}-w_{2 \tilde{B}}^{U}\right)^{2} .
\end{array}\right]}=0
$$

, which implies that

$$
a_{1}^{L}=b_{1}^{L}, a_{2}^{L}=b_{2}^{L}, a_{3}^{L}=b_{3}^{L}, a_{4}^{L}=b_{4^{\prime}}^{L} a_{1}^{U}=b_{1}^{U}, a_{2}^{U}=b_{2}^{U}, a_{3}^{U}=b_{3}^{U}, a_{4}^{U}=b_{4}^{U}, w_{1 \tilde{A}}^{L}=w_{1 \tilde{B}^{\prime}}^{L}, w_{2 B}^{L}=w_{2 \tilde{A}^{\prime}}^{L} w_{1 \tilde{A}}^{U}=w_{1 B^{\prime}}^{U} \text { and } w_{2 \tilde{A}}^{U}=w_{2 B^{\prime}}^{U} \text { then } \tilde{A}=\tilde{B}
$$

Wu and Mendel [29] proposed 32 words for computing with words. They can be grouped into three classes. Class one: small-sounding words (none to very little, teeny-weeny, a smidgen, tiny, very small, very little, a bit, little, low amount, small, and somewhat small). Class two: medium-sounding words (some, some to moderate, moderate amount, fair amount, medium, modest amount, and good amount). Class three: large-sounding words (sizeable, quite a bit, considerable amount, substantial amount, a lot, high amount, very sizeable, large, humongous amount, huge amount, very high amount, extreme amount, and maximum amount). For the corresponding IT2FSs of these words the reader is referred to $\mathrm{Wu}$ and Mendel [29] or Heidarzade et al. [13].

The performance of the extended vertex method is compared with the performance of the signed distance and the method of Heidarzade et al. [13]. Both the signed distance and the method of Heidarzade et al. [13] for trapezoidal IT2FSs are restricted to normalized IT2FSs with equal membership values i.e. $w_{1}^{L}=w_{2}^{L}$ and $w_{1}^{U}=w_{2}^{U}$. The extended vertex method computes the distance between
From Table 1, it is obvious that the distances obtained by the method of Heidarzade et al. [13] are in an increasing order. As for the distances obtained by the signed distance, they are in an increasing order except for the words very small and very little, little and low amount, and substantial amount and a lot. Regarding the extended vertex method, the distances are also in an increasing order except for the words fair amount and medium, substantial amount and a lot, and very sizeable and large. In any case, the maximum difference was 0.2 on the 0 to 10 scale. This is acceptable in fuzzy sets as these words are quite equivalent when defuzzified, and the order may differ according to the used defuzzification technique. The results indicate the extended vertex method is appropriate for measuring the distances between IT2FSs.

Regarding the computational complexity, the extended vertex method has the least processing time. When implemented using MatLab on a PC (Intel(R) Core(TM) i3-6100 CPU@2.3 GHz), the processing time was as follows. The signed distance takes $1.8 \times 10^{-5} \mathrm{~s}$. The method of Heidarzade et al. [13] takes $3.3 \times 10^{-5} \mathrm{~s}$, using the minimum 
Table 1 The distance between the first element and the other elements

\begin{tabular}{|c|c|c|c|}
\hline Distance & Signed distance & $\begin{array}{l}\text { Heidarzade et al. } \\
\text { method [13] }\end{array}$ & $\begin{array}{l}\text { Extended ver- } \\
\text { tex method }\end{array}$ \\
\hline D (none to very little, teeny-weeny) & 0.0072 & 0.0089 & 0.1879 \\
\hline D (none to very little, a smidgen) & 0.0153 & 0.0111 & 0.2374 \\
\hline $\mathrm{D}$ (none to very little, tiny) & 0.0261 & 0.0120 & 0.2462 \\
\hline D (none to very little, very small) & 0.0443 & 0.0208 & 0.3067 \\
\hline D (none to very little, very little) & 0.0441 & 0.0210 & 0.3155 \\
\hline D (none to very little, a bit) & 0.3108 & 0.1321 & 1.4466 \\
\hline D (none to very little, little) & 0.3748 & 0.1635 & 1.7866 \\
\hline D (none to very little, low amount) & 0.3726 & 0.1640 & 1.8546 \\
\hline D (none to very little, small) & 0.4171 & 0.1865 & 2.1266 \\
\hline D (none to very little, somewhat small) & 0.4791 & 0.2176 & 2.3793 \\
\hline D (none to very little, some) & 0.7253 & 0.3382 & 3.7383 \\
\hline D (none to very little, some to moderate) & 0.8677 & 0.4137 & 4.4649 \\
\hline D (none to very little, moderate amount) & 0.9331 & 0.4444 & 4.5810 \\
\hline D (none to very little, fair amount) & 0.9852 & 0.4723 & 4.9908 \\
\hline D (none to very little, medium) & 0.9874 & 0.4733 & 4.7911 \\
\hline D (none to very little, modest amount) & 1.0363 & 0.4944 & 5.0517 \\
\hline D (none to very little, good amount) & 1.2577 & 0.6060 & 6.2784 \\
\hline D (none to very little, sizeable) & 1.3947 & 0.6754 & 6.9856 \\
\hline D (none to very little, quite a bit) & 1.3947 & 0.6754 & 6.9856 \\
\hline D (none to very little, considerable amount) & 1.4189 & 0.6892 & 7.1535 \\
\hline D (none to very little, substantial amount) & 1.5543 & 0.7540 & 7.7687 \\
\hline D (none to very little, a lot) & 1.5538 & 0.7542 & 7.7505 \\
\hline D (none to very little, high amount) & 1.5645 & 0.7602 & 7.8420 \\
\hline D (none to very little, very sizeable) & 1.5811 & 0.7713 & 8.0113 \\
\hline D (none to very little, large) & 1.5836 & 0.7716 & 7.9105 \\
\hline D (none to very little, very large) & 1.8708 & 0.9818 & 9.1176 \\
\hline D (none to very little, humongous amount) & 1.8852 & 0.8982 & 9.1715 \\
\hline D (none to very little, huge amount) & 1.8991 & 0.9093 & 9.2410 \\
\hline D (none to very little, very high amount) & 1.9051 & 0.9105 & 9.2567 \\
\hline D (none to very little, extreme amount) & 1.9101 & 0.9244 & 9.3083 \\
\hline D (none to very little, maximum amount) & 1.9303 & 0.9309 & 9.4422 \\
\hline
\end{tabular}

number of embedded T1FSs (only the upper and lower membership functions), in practice large number of embedded T1FSs are used, e.g., 10 or 20 . The extended vertex method takes $1.6 \times 10^{-5} \mathrm{~s}$. Since calculating the distance between IT2FSs is repeated $2 m n$ times ( $m$ criteria and $n$ variables) in IT2F-TOPSIS, then the extended vertex method reduces the computations.

\section{The proposed TOPSIS}

TOPSIS was first introduced by Hwang and Yoon [14] for real-valued data. Later, Chen [2] extended the method to the fuzzy environment using T1FSs. Chen and Lee [5] modified the technique to use IT2FSs. Recent research has been devoted to the fuzzy extension of TOPSIS, but only a few studies handled IT2FSs [8].
In this section, The TOPSIS method is modified. First, the PIS and NIS proposed by Rashid et al. [23] for IVFSs are extended to IT2FSs. Then, the extended vertex method is used to calculate the distance between the alternatives and the ideal solutions.

For a MAGDM problem, let $X=\left\{x_{1}, x_{2}, \ldots, x_{n}\right\}$ be the set of $n$ alternatives and $F=\left\{f_{1}, f_{2}, \ldots, f_{m}\right\}$ be the set of $m$ attributes in the presence of $k$ decision makers $D_{1}, D_{2}, \ldots, D_{k}$. The set of attributes " $F$ " is divided into two sets, the set of benefit attributes " $F_{b}$ " and the set of cost attributes " $F_{c}$ " such that $F_{b} \cap F_{c}=\emptyset$. It is assumed that the data used is normalized i.e. the fuzzy sets lies in the interval $[0,1]$.

Step 1: Constructing the fuzzy decision matrix and the average decision matrix 


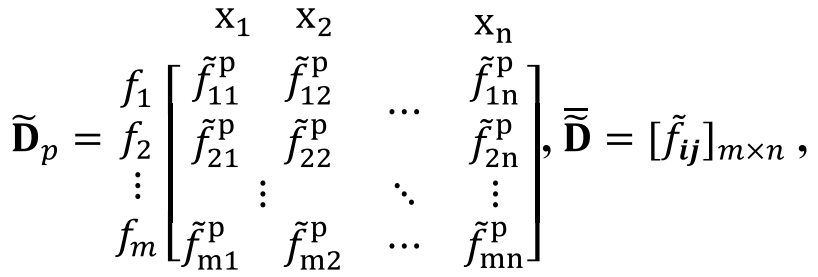

where $\quad \tilde{f}_{i j}=\left(\frac{\tilde{f}_{i j}^{1} \oplus \tilde{f}_{i j}^{2} \oplus \cdots \oplus \tilde{f}_{i j}^{k}}{k}\right)$ is an IT $2 \mathrm{FS}$, $1 \leq i \leq m, 1 \leq j \leq n$ and $1 \leq p \leq k$.

Step 2: Constructing the weighting matrix and the average weighting matrix,

$$
\begin{array}{rlll}
f_{1} & f_{2} & & f_{m} \\
\widetilde{\mathbf{W}}_{p}=\left[\begin{array}{llll}
\widetilde{\mathbf{w}}_{1}^{p} & \widetilde{\mathbf{w}}_{2}^{p} & \ldots & \widetilde{\mathbf{w}}_{m}^{p}
\end{array}\right], \quad \overline{\widetilde{\mathbf{W}}}=\left[\widetilde{\mathbf{w}}_{\boldsymbol{i}}\right]_{1 \times m}
\end{array}
$$

where $\tilde{w}_{\boldsymbol{i}}=\left(\frac{\tilde{w}_{i}^{1} \oplus \tilde{w}_{i}^{2} \oplus \cdots \oplus \tilde{\mathrm{w}}_{i}^{k}}{k}\right)$ is an IT2FS, $1 \leq i \leq m$ and $1 \leq p \leq k$.

Step 3: Constructing the normalized weighted decision matrix

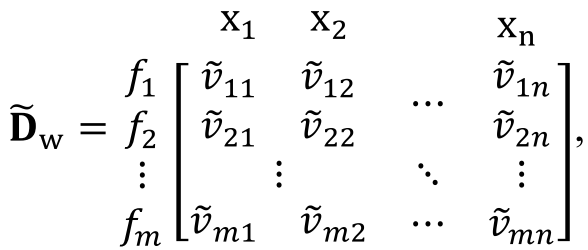

where $\tilde{v}_{i j}=\tilde{\mathrm{w}}_{i} \otimes \tilde{f}_{i j}, 1 \leq i \leq m$ and $1 \leq j \leq n$.

Step 4: Defining the fuzzy PIS $\left(\tilde{\mathbf{V}}^{+}\right)$and the fuzzy NIS solution $\left(\tilde{\mathbf{V}}^{-}\right)$where,

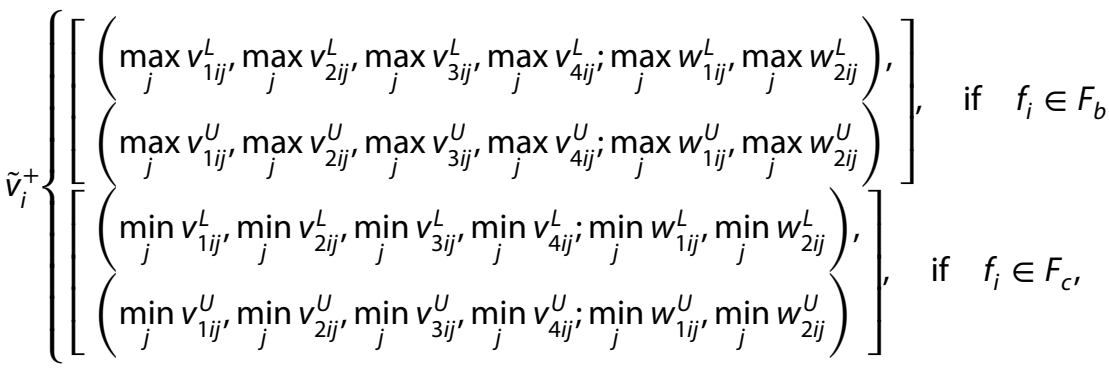

$$
\mathbf{V}^{+}=\left[\begin{array}{llll}
\tilde{v}_{1}^{+} & \tilde{v}_{2}^{+} & \ldots & \tilde{v}_{m}^{+}
\end{array}\right]
$$

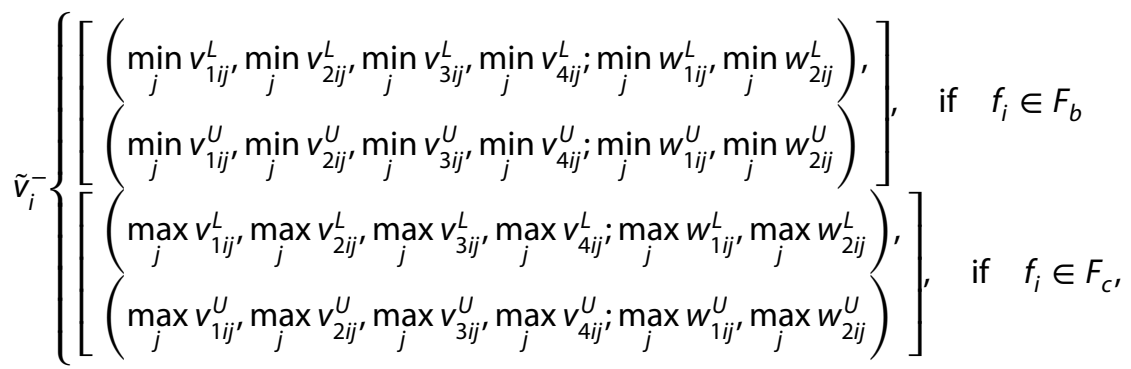

$$
\mathbf{V}^{-}=\left[\begin{array}{llll}
\tilde{v}_{1}^{-} & \tilde{v}_{2}^{-} & \ldots & \tilde{v}_{m}^{-}
\end{array}\right] .
$$

Step 5: Constructing the ideal separation matrix $\mathbf{S}^{+}$and the anti-ideal separation matrix $\mathbf{S}^{-}$using the extended vertex method for distance measure.

$$
\begin{aligned}
& x_{1} \quad x_{2} \\
& \mathbf{S}^{+}=\left[\begin{array}{llll}
\sum_{i=1}^{m} d\left(\tilde{v}_{i 1}, v_{i}^{+}\right) \quad \sum_{i=1}^{m} d\left(\tilde{v}_{i 2}, v_{i}^{+}\right) \quad \ldots \sum_{i=1}^{m} d\left(\tilde{v}_{i n}, v_{i}^{+}\right)
\end{array}\right],
\end{aligned}
$$

and

$$
\mathbf{S}^{-}=\left[\begin{array}{cccc}
\sum_{1}^{m} d\left(\tilde{v}_{i 1}, v_{i}^{-}\right) & \sum_{i=1}^{m} d\left(\tilde{v}_{i 2}, v_{i}^{-}\right) & \ldots & \sum_{i=1}^{m} d\left(\tilde{v}_{i n}, v_{i}^{-}\right)
\end{array}\right] .
$$


Step 6: Calculating the relative degree of closeness of each alternative to the ideal solution and ranking.

$$
R\left(x_{j}\right)=\frac{S^{-}\left(x_{j}\right)}{S^{+}\left(x_{j}\right)+S^{-}\left(x_{j}\right)}
$$

\section{Examples}

In this section, two examples are solved. The first example is due to Rashid et al. [23], the second example is due Chen and Hong [3]. The results of the proposed IT2F-TOPSIS are compared with their results.

Example 1 A manufacturing company intends to purchase robots for material-handling. The decision makers 1,2 , and 3 evaluates three robots on six attributes: man-machine interface $\left(f_{1}\right)$, programming flexibility $\left(f_{2}\right)$, vendor's service contract $\left(f_{3}\right)$, purchase cost $\left(f_{4}\right)$, load capacity $\left(f_{5}\right)$, and positioning accuracy $\left(f_{6}\right)$. The benefit attributes are $f_{1}, f_{2}, f_{3}$ and $f_{5}$. The cost attributes are $f_{4}$ and $f_{6}$. Some of the attributes are subjective $f_{1}, f_{2}$ and $f_{3}$. The other three attributes are objective $f_{4}, f_{5}$ and $f_{6}$. Let $X=\left\{x_{1}, x_{2}, x_{3}\right\}$ be the set of alternatives, and $F=\left\{f_{1}, f_{2}, f_{3}, f_{4}, f_{5}, f_{6}\right\}$ be the set of attributes. The decision makers use two sets of linguistic terms: the weighting set $W=\operatorname{Very} \operatorname{Low}(\mathrm{VL}), \operatorname{Low}(\mathrm{L}), \operatorname{Medium}(\mathrm{M}), \operatorname{High}(\mathrm{H})$, Very High $(\mathrm{VH})$ and the rating set $R=$ Very Poor(VP), Poor(P), Fair(F), Good(G), Very Good(VG). The objective attributes are denoted by $\mathrm{O}_{i}^{j}$ in the decision matrices. To summarize, only the elements of the normalized weighted decision matrix are given. For the interpretation of linguistic variables in terms of IT2FSs and complete details, see Rashid et al. [23].

Step 1: a) Constructing the decision matrices, and the average decision matrix

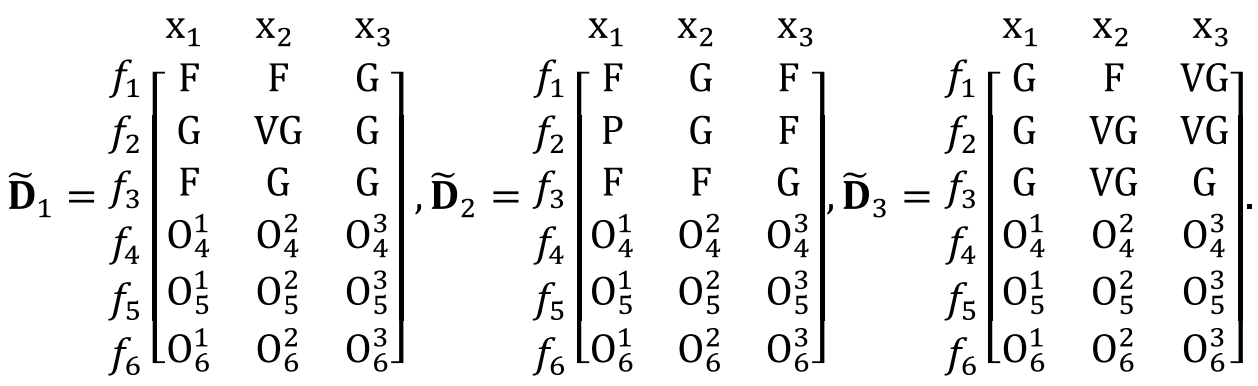

$$
\begin{aligned}
& \begin{array}{lll}
\mathrm{x}_{1} & \mathrm{x}_{2} & \mathrm{x}_{3}
\end{array} \\
& f_{1}\left[\begin{array}{lll}
\tilde{f}_{11} & \tilde{f}_{12} & \tilde{f}_{13}
\end{array}\right] \\
& f_{2} \begin{array}{lll}
\tilde{f}_{21} & \tilde{f}_{22} & \tilde{f}_{23}
\end{array} \\
& \overline{\widetilde{\mathbf{D}}}=f_{3} \mid \begin{array}{ccc}
\tilde{f}_{31} & \tilde{f}_{32} & \tilde{f}_{33} \\
\tilde{f}_{41} & \tilde{f}_{42} & \tilde{f}_{43}
\end{array} \text {. } \\
& f_{4} \tilde{f}_{41} \quad \tilde{f}_{42} \quad \tilde{f}_{43} \\
& f_{5}\left[\begin{array}{lll}
\tilde{f}_{51} & \tilde{f}_{52} & \tilde{f}_{53}
\end{array}\right. \\
& f_{6}\left[\begin{array}{lll}
\tilde{f}_{61} & \tilde{f}_{62} & \tilde{f}_{63}
\end{array}\right]
\end{aligned}
$$

Step 2: a) Constructing the weighting matrices, and the average weighting matrix

$$
\begin{aligned}
& \begin{array}{llllllllllllllllll}
f_{1} & f_{2} & f_{3} & f_{4} & f_{5} & f_{6} & f_{1} & f_{2} & f_{3} & f_{4} & f_{5} & f_{6} & f_{1} & f_{2} & f_{3} & f_{4} & f_{5} & f_{6}
\end{array} \\
& \widetilde{\mathbf{W}}_{1}=\left[\begin{array}{llllll}
\mathrm{H} & \mathrm{VH} & \mathrm{M} & \mathrm{M} & \mathrm{VH} & \mathrm{VH}
\end{array}\right], \widetilde{\mathbf{W}}_{2}=\left[\begin{array}{llllllllll}
\text { VH } & \mathrm{H} & \mathrm{L} & \mathrm{M} & \mathrm{VH} & \mathrm{H}
\end{array}\right], \widetilde{\mathbf{w}}_{3}=\left[\begin{array}{llllll}
\text { VH } & \text { VH } & \text { M } & \text { M } & \mathrm{H} & \mathrm{H}
\end{array}\right] . \\
& \begin{array}{llllll}
f_{1} & f_{2} & f_{3} & f_{4} & f_{5} & f_{6}
\end{array} \\
& \overline{\widetilde{\mathbf{W}}}=\left[\begin{array}{llllll}
\widetilde{w}_{1} & \widetilde{w}_{2} & \widetilde{W}_{3} & \widetilde{w}_{4} & \widetilde{w}_{5} & \widetilde{w}_{6}
\end{array}\right] .
\end{aligned}
$$

Step 3: Constructing the weighted normalized decision matrix 


$\left.\begin{array}{r|rll} & \mathrm{x}_{1} & \mathrm{x}_{2} & \mathrm{x}_{3} \\ f_{1} & \tilde{v}_{11} & \tilde{v}_{12} & \tilde{v}_{13} \\ f_{2} & \tilde{v}_{21} & \tilde{v}_{22} & \tilde{v}_{23} \\ f_{3} & \tilde{v}_{31} & \tilde{v}_{32} & \tilde{v}_{33} \\ f_{4} & \tilde{v}_{41} & \tilde{v}_{42} & \tilde{v}_{43} \\ f_{5} & \tilde{v}_{51} & \tilde{v}_{52} & \tilde{v}_{53} \\ f_{6} & \tilde{v}_{61} & \tilde{v}_{62} & \tilde{v}_{63}\end{array}\right]$.

Step 4: Define the fuzzy positive ideal solution

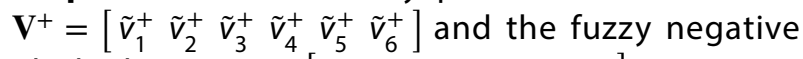
ideal solution $\mathbf{V}^{-}=\left[\begin{array}{llllll}\tilde{v}_{1}^{-} & \tilde{v}_{2}^{-} & \tilde{v}_{3}^{-} & \tilde{v}_{4}^{-} & \tilde{v}_{5}^{-} & \tilde{v}_{6}^{-}\end{array}\right]$.

$\tilde{v}_{11}=[(0.5408,0.6094,0.7157,0.7516 ; 0.8,0.8)(0.4464,0.5577,0.7727,0.8577 ; 1,1)]$,

$\tilde{v}_{12}=[(0.5408,0.6094,0.7157,0.7516 ; 0.8,0.8)(0.4464,0.5577,0.7727,0.8577 ; 1,1)]$,

$\tilde{v}_{13}=[(0.7264,0.7981,0.8818,0.9080 ; 0.8,0.8)(0.6446,0.7564,0.9287,0.9900 ; 1,1)]$,

$\tilde{v}_{21}=[(0.4965,0.5469,0.6216,0.6484 ; 0.8,0.8)(0.4285,0.5104,0.6619,0.7233 ; 1,1)]$,

$\tilde{v}_{22}=[(0.8046,0.8705,0.9244,0.9390 ; 0.8,0.8)(0.7470,0.8426,0.9569,0.9900 ; 1,1)]$,

$\tilde{v}_{23}=[(0.6408,0.7040,0.7778,0.8010 ; 0.8,0.8)(0.5704,0.6673,0.8193,0.8733 ; 1,1)]$,

$\tilde{v}_{31}=[(0.1622,0.2019,0.2772,0.3082 ; 0.8,0.8)(0.1059,0.1686,0.3192,0.3978 ; 1,1)]$,

$\tilde{v}_{32}=[(0.2180,0.2644,0.3416,0.3727 ; 0.8,0.8)(0.1534,0.2286,0.3837,0.4590 ; 1,1)]$,

$\tilde{v}_{33}=[(0.2399,0.2870,0.3755,0.4108 ; 0.8,0.8)(0.1682,0.2465,0.4236,0.5100 ; 1,1)]$,

$\tilde{v}_{41}=[(0.3649,0.4170,0.4967,0.5262 ; 0.8,0.8)(0.2897,0.3737,0.5378,0.6048 ; 1,1)]$,

$\tilde{v}_{42}=[(0.3798,0.4331,0.5181,0.5510 ; 0.8,0.8)(0.2977,0.3841,0.5648,0.6358 ; 1,1)]$,

$\tilde{v}_{43}=[(0.3880,0.4393,0.5295,0.5632 ; 0.8,0.8)(0.3049,0.3924,0.5774,0.6500 ; 1,1)]$,

$\tilde{v}_{51}=[(0.8324,0.8747,0.9198,0.9363 ; 0.8,0.8)(0.7938,0.8474,0.9546,0.9900 ; 1,1)]$,

$\tilde{v}_{52}=[(0.7551,0.7944,0.8278,0.8436 ; 0.8,0.8)(0.7194,0.7693,0.8610,0.8852 ; 1,1)]$,

$\tilde{v}_{53}=[(0.7466,0.7855,0.8278,0.8436 ; 0.8,0.8)(0.7111,0.7605,0.8797,0.9043 ; 1,1)]$,

$\tilde{v}_{61}=[(0.6203,0.6705,0.7673,0.8137 ; 0.8,0.8)(0.5563,0.6047,0.8608,0.9800 ; 1,1)]$,

$\tilde{v}_{62}=[(0.4926,0.5282,0.5865,0.6238 ; 0.8,0.8)(0.4270,0.4703,0.6528,0.7000 ; 1,1)]$,

$\tilde{v}_{63}=[(0.4785,0.5127,0.5580,0.5776 ; 0.8,0.8)(0.4157,0.4576,05916,0.6322 ; 1,1)]$

$\tilde{v}_{1}^{+}=[(0.7264,0.7981,0.8818,0.9080 ; 0.8,0.8)(0.6466,0.7564,0.9287,0.9900 ; 1,1)]$,

$\tilde{v}_{2}^{+}=[(0.8046,0.8705,0.9244,0.9390 ; 0.8,0.8)(0.7470,0.8426,0.9569,0.9900 ; 1,1)]$,

$\tilde{v}_{3}^{+}=[(0.2399,0.2870,0.3755,0.4108 ; 0.8,0.8)(0.1682,0.2465,0.4236,0.5100 ; 1,1)]$,

$\tilde{v}_{4}^{+}=[(0.3649,0.4170,0.4967,0.5262 ; 0.8,0.8)(0.2897,0.3737,0.5378,0.6048 ; 1,1)]$,

$\tilde{v}_{5}^{+}=[(0.8324,0.8747,0.9198,0.9363 ; 0.8,0.8)(0.7938,0.8474,0.9546,0.9900 ; 1,1)]$,

$\tilde{v}_{6}^{+}=[(0.4785,0.5127,0.5580,0.5776 ; 0.8,0.8)(0.4157,0.4576,0.5916,0.6322 ; 1,1)]$,

$\tilde{v}_{1}^{-}=[(0.5408,0.6094,0.7157,0.7516 ; 0.8,0.8)(0.4464,0.5577,0.7727,0.8577 ; 1,1)]$,

$\tilde{v}_{2}^{-}=[(0.4965,0.5469,0.6216,0.6484 ; 0.8,0.8)(0.4285,0.5104,0.6619,0.7233 ; 1,1)]$,

$\tilde{v}_{3}^{-}=[(0.1622,0.2019,0.2772,0.3082 ; 0.8,0.8)(0.1059,0.1686,0.3192,0.3978 ; 1,1)]$,

$\tilde{v}_{4}^{-}=[(0.3880,0.4393,0.5295,0.5632 ; 0.8,0.8)(0.3049,0.3924,0.5774,0.6500 ; 1,1)]$,

$\tilde{v}_{5}^{-}=[(0.7466,0.7855,0.8275,0.8436 ; 0.8,0.8)(0.7111,0.7605,0.8610,0.8852 ; 1,1)]$,

$\tilde{v}_{6}^{-}=[(0.6203,0.6705,0.7673,0.8137 ; 0.8,0.8)(0.5563,0.6047,0.8606,0.9800 ; 1,1)]$. 
Step 5: Constructing the ideal and the anti-ideal separation matrices using the extended vertex method for distance measure.
From the results, $R\left(\mathrm{x}_{3}\right)>R\left(\mathrm{x}_{2}\right)>R\left(\mathrm{x}_{1}\right)$, the ranking is $\mathrm{x}_{3}>\mathrm{x}_{2}>\mathrm{x}_{1}$. Then, $\mathrm{x}_{3}$ is the best alternative. The result coincides with the results of Rashid et al. [23].

$$
\begin{aligned}
& \sum_{i=1}^{m} d\left(\tilde{v}_{i 1}, v_{i}^{+}\right)=0.1745+0.3053+0.0915+0+0+0.2178=0.7891 \\
& \sum_{i=1}^{m} d\left(\tilde{v}_{i 2}, v_{i}^{+}\right)=0.1745+0+0.0323+0.0203+.0872+.0388=0.3531 \\
& \sum_{i=1}^{m} d\left(\tilde{v}_{i 3}, v_{i}^{+}\right)=0+0.1539+0+0.0306+0.0864+0=0.2709
\end{aligned}
$$

Then

$$
\mathbf{S}^{+}=\left[\begin{array}{ccc}
\mathrm{x}_{1} & \mathrm{x}_{2} & \mathrm{x}_{3} \\
0.7891 & 0.3531 & 0.2709] .
\end{array}\right.
$$

Similarly
Example 2 A company wants to hire a system analyst. Three decision makers $D_{1}, D_{2}$, and $D_{3}$ will count on two attributes to rate the applicants: emotional steadiness $(f 1)$ and oral communication skills $(f 2)$. The decision makers use two sets of linguistic terms: the weighting set $W=\{\operatorname{Very} \operatorname{Low}(\mathrm{VL}), \operatorname{Low}(\mathrm{L}), \operatorname{Medium}-\operatorname{low}(\mathrm{ML}), \operatorname{Medium}(\mathrm{M})$,

$$
\begin{aligned}
& \sum_{i=1}^{m} d\left(\tilde{v}_{i 1}, v_{i}^{-}\right)=0+0+0+0.0306+0.0864+0=0.1170 \\
& \sum_{i=1}^{m} d\left(\tilde{v}_{i 2}, v_{i}^{-}\right)=0+0.3053+0.0602+0.0104+0.0112+0.1809=0.5680 \\
& \sum_{i=1}^{m} d\left(\tilde{v}_{i 3}, v_{i}^{-}\right)=0.1745+0.1522+0.0915+0+0+0.2178=0.6360 .
\end{aligned}
$$

Then

$$
\mathbf{S}^{-}=\left[\begin{array}{ccc}
\mathrm{x}_{1} & \mathrm{x}_{2} & \mathrm{x}_{3} \\
0.1170 & 0.5680 & 0.6360
\end{array}\right] .
$$

Step 6: Calculating the relative degree of closeness of each alternative to the fuzzy ideal solutions.

$$
\begin{aligned}
& R\left(\mathrm{x}_{1}\right)=\frac{0.1170}{0.1170+0.7891}=0.1291 \\
& R\left(\mathrm{x}_{2}\right)=\frac{0.5680}{0.5680+0.3531}=0.6167 \\
& R\left(\mathrm{x}_{3}\right)=\frac{0.6360}{0.6360+0.2709}=0.7013
\end{aligned}
$$

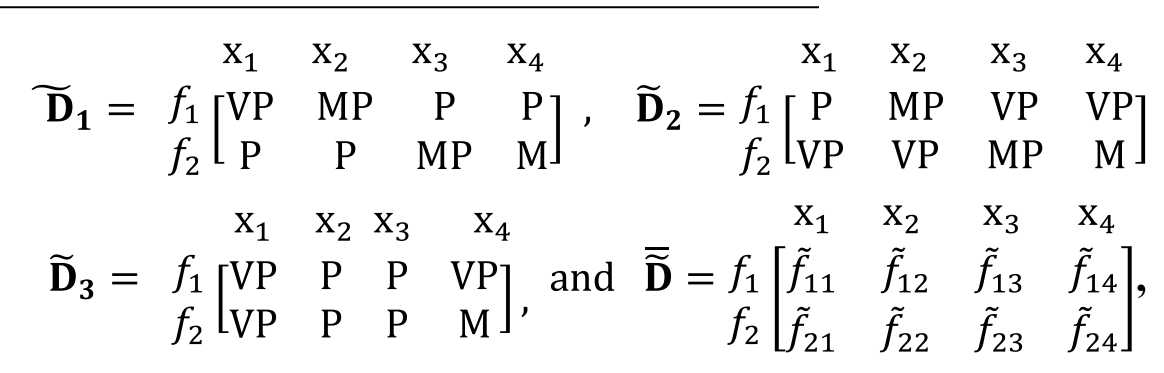

Medium - High (MH), High(H), Very High (VH) \}, and the rating set

$R=\{$ Very Poor(VP), Poor(P), Medium Poor(MP),

Medium(M), Medium Good(MG),

$\operatorname{Good}(\mathrm{G})$, Very Good(VG)\}. For the details of the IT2FSs representation of the linguistic ratings and the attributes' weights, the reader is referred to Chen and Hong [3]. For comparison, the normalized ratings are used directly in the decision matrices as given by Chen and Hong [3].

Step 1: Constructing the decision matrices and the average decision matrix 
where

$\tilde{f}_{11}=[(0,0.03,0.03,0.17 ; 1,1)(0,0.03,0.03,0.17 ; 1,1)]$,

$\tilde{f}_{12}=[(0.07,0.23,0.23,0.43 ; 1,1)(0.07,0.23,0.23,0.43 ; 1,1)]$,

$\tilde{f}_{13}=[(0,0.07,0.07,0.1 ; 1,1)(0,0.07,0.07,0.1 ; 1,1)]$,

$\tilde{f}_{14}=[(0,0.07,0.07,0.1 ; 1,1)(0,0.07,0.07,0.1 ; 1,1)]$,

$\tilde{f}_{21}=[(0,0.03,0.03,0.17 ; 1,1)(0,0.03,0.03,0.17 ; 1,1)]$,

$\tilde{f}_{22}=[(0,0.07,0.07,0.1 ; 1,1)(0,0.07,0.07,0.1 ; 1,1)]$,

$\tilde{f}_{23}=[(0.07,0.23,0.23,0.43 ; 1,1)(0.07,0.23,0.23,0.43 ; 1,1)]$,

$\tilde{f}_{24}=[(0.3,0.5,0.5,0.7 ; 1,1)(0.3,0.5,0.5,0.7 ; 1,1)]$. $\tilde{w}_{1}=[(0.63,0.83,0.83,0.97 ; 1,1)(0.63,0.83,0.83,0.97 ; 1,1)]$,

$\tilde{w}_{2}=[(0.63,0.83,0.83,0.97 ; 1,1)(0.63,0.83,0.83,0.97 ; 1,1)]$.

Step 3: Constructing the average weighted decision matrix.

$$
\begin{aligned}
& \begin{array}{llll}
\mathrm{x}_{1} & \mathrm{x}_{2} & \mathrm{x}_{3} & \mathrm{x}_{4}
\end{array} \\
& \widetilde{\mathbf{D}}_{\mathrm{w}}=f_{1}\left[\begin{array}{llll}
\tilde{v}_{11} & \tilde{v}_{12} & \tilde{v}_{13} & \tilde{v}_{14} \\
\tilde{v}_{21} & \tilde{v}_{22} & \tilde{v}_{23} & \tilde{v}_{24}
\end{array}\right],
\end{aligned}
$$

where,

$\tilde{V}_{11}=[(0,0.0249,0.0249,0.1649 ; 1,1)(0,0.0249,0.0249,0.1649 ; 1,1)]$,

$\tilde{v}_{12}=[(0.0441,0.1909,0.1909,0.4171 ; 1,1)(0.0441,0.1909,0.1909,0.4171 ; 1,1)]$,

$\tilde{v}_{13}=[(0,0.0581,0.0581,0.0970 ; 1,1)(0,0.0581,0.0581,0.0970 ; 1,1)]$,

$\tilde{v}_{14}=[(0,0.0581,0.0581,0.0970 ; 1,1)(0,0.0581,0.0581,0.0970 ; 1,1)]$,

$\tilde{v}_{21}=[(0,0.0249,0.0249,0.1649 ; 1,1)(0,0.0249,0.0249,0.1649 ; 1,1)]$,

$\tilde{v}_{22}=[(0,0.0581,0.0581,0.0970 ; 1,1)(0,0.0581,0.0581,0.0970 ; 1,1)]$,

$\tilde{v}_{23}=[(0.0441,0.1909,0.1909,0.4171 ; 1,1)(0.0441,0.1909,0.1909,0.4171 ; 1,1)]$,

$\tilde{v}_{24}=[(0.1890,0.4150,0.4150,0.6790 ; 1,1)(0.1890,0.4150,0.4150,0.6790 ; 1,1)]$.

Step 4: Define the fuzzy positive ideal solution $\mathbf{V}^{+}=\left[\begin{array}{ll}\tilde{v}_{1}^{+} & \tilde{v}_{2}^{+}\end{array}\right]$and the fuzzy negative ideal solution $\mathbf{V}^{-}=\left[\begin{array}{ll}\tilde{v}_{1}^{-} & \tilde{v}_{2}^{-}\end{array}\right]$.

$\tilde{v}_{1}^{+}=[(0.0441,0.1909,0.1909,0.4171 ; 1,1)(0.0441,0.1909,0.1909,0.4171 ; 1,1)]$,

$\tilde{v}_{2}^{+}=[(0.1890,0.4150,0.4150,0.6790 ; 1,1)(0.1890,0.4150,0.4150,0.6790 ; 1,1)]$.

$\tilde{v}_{1}^{-}=[(0,0.0249,0.0249,0.0970 ; 1,1)(0,0.0249,0.0249,0.0970 ; 1,1)]$,

$\tilde{v}_{2}^{-}=[(0,0.0249,0.0249,0.0970 ; 1,1)(0,0.0249,0.0249,0.0970 ; 1,1)]$.

Step 2: a) Constructing the weighting matrices and the average weighting matrix.

$$
\begin{aligned}
& \begin{array}{llllll}
f_{1} & f_{2} & f_{1} & f_{2} & f_{1} & f_{2}
\end{array} \\
& \widetilde{\mathbf{W}}_{1}=\left[\begin{array}{ll}
\mathrm{H} & \mathrm{H}
\end{array}\right], \widetilde{\mathbf{W}}_{2}=\left[\begin{array}{ll}
\mathrm{MH} & \mathrm{MH}
\end{array}\right], \widetilde{\mathbf{W}}_{3}=\left[\begin{array}{ll}
\mathrm{H} & \mathrm{H}
\end{array}\right], \\
& f_{1} \quad f_{2} \\
& \overline{\widetilde{\mathbf{W}}}=\left[\begin{array}{ll}
\widetilde{w}_{1} & \widetilde{w}_{2}
\end{array}\right],
\end{aligned}
$$

where
Step 5: Constructing the ideal and the anti-ideal separation matrices.

Then 


$$
\begin{aligned}
& \begin{array}{llll}
\mathrm{x}_{1} & \mathrm{x}_{2} & \mathrm{X}_{3} & \mathrm{x}_{4}
\end{array} \\
& S^{+}=\left[\begin{array}{llll}
0.5624 & 0.3966 & 0.4049 & 0.1869
\end{array}\right] . \\
& \sum_{i=1}^{m} d\left(\tilde{v}_{i 1}, v_{i}^{-}\right)=0.0339+0.0339=0.0678, \\
& \sum_{i=1}^{m} d\left(\tilde{v}_{i 2}, v_{i}^{-}\right)=0.1997+0.0235=0.2232 \text {, } \\
& \sum_{i=1}^{m} d\left(\tilde{v}_{i 3}, v_{i}^{-}\right)=0.0235+0.1997=0.2232, \\
& \sum_{i=1}^{m} d\left(\tilde{v}_{i 4}, v_{i}^{-}\right)=0.0235+0.4119=0.4354 \text {. }
\end{aligned}
$$

Then

$$
\left.\mathbf{S}^{-}=\begin{array}{clll}
\mathrm{x}_{1} & \mathrm{x}_{2} & \mathrm{x}_{3} & \mathrm{x}_{4} \\
0.0678 & 0.2232 & 0.2232 & 0.4354
\end{array}\right] .
$$

Step 6: Calculating the relative degree of closeness of each alternative to the ideal solution. average weighted decision matrix where $\tilde{v}_{12}=\tilde{v}_{23}$ and $\tilde{v}_{13}=\tilde{v}_{22}$.

On the other hand, the influence of the ideal solutions was not taken into consideration. In this example, the positive ideal solution for the emotional steadiness is different from that of the oral communication skills, i.e. $\tilde{v}_{1}^{+} \neq \tilde{v}_{2}^{+}$. Consequently, the distance between $\tilde{v}_{12}$ and $\tilde{v}_{1}^{+}$is different from the distance between $\tilde{v}_{23}$ and $\tilde{v}_{2}^{+}$. Similarly, the distance between $\tilde{v}_{13}$ and $\tilde{v}_{1}^{+}$is different from the distance between $\tilde{v}_{22}$ and $\tilde{v}_{2}^{+}$. Therefore, this difference has an impact on the ranking and lead to the preference of the second alternative over the third one. This asserts that early defuzzification may affect the results and give an incorrect preference. In addition, it emphasizes on the role of the defined ideal solutions in ranking.

When proposing the PIS and the NIS, some researchers use the absolute ideal solutions $\left\{v^{+}=(1,1,1,1 ; 1,1)\right.$ and $\left.v^{-}=(0,0,0,0 ; 1,1)\right\}$, which represent the perfect PIS and NIS that can be attained. Other researchers use the relative ideal solutions, as in the proposed IT2F-TOPSIS, which are related to the performance of the available alternatives

$$
\begin{array}{ll}
R\left(\mathrm{x}_{1}\right)=\frac{0.0678}{0.0678+0.5624}=0.1076, \quad R\left(\mathrm{x}_{2}\right)=\frac{0.2232}{0.2232+0.3966}=0.3601, \\
R\left(\mathrm{x}_{3}\right)=\frac{0.2232}{0.2332+0.4049}=0.3554, \quad R\left(\mathrm{x}_{4}\right)=\frac{0.4354}{0.4354+0.1809}=0.6997 .
\end{array}
$$

From the results, $R\left(\mathrm{x}_{4}\right)>R\left(\mathrm{x}_{2}\right)>R\left(\mathrm{x}_{3}\right)>R\left(\mathrm{x}_{1}\right)$. Then, the ranking is $x_{4}>x_{2}>x_{3}>x_{1}$. This ranking coincides with the ranking of Chen and Lee [5]. The ranking of Chen and Hong [3] is $x_{4}>x_{2}=x_{3}>x_{1}$. Therefore, the best and the worst alternative are the same as their result. However, the second alternative is not equivalent to the third. The second alternative is better than the third with a slight difference.

\section{Discussion}

According to Chen and Hong [3], the second and third alternatives should be equally preferred, and this was supported by the results of their proposed IT2F-TOPSIS. As justified by Chen and Hong [3], this is due to the decision makers' similar evaluations of the alternatives with respect to the given attributes together with the equivalence in weights. The decision makers' evaluations of the second attribute with respect to the emotional steadiness ( $f 1)$ are $\{M P, M P, P\}$, and for the oral communication skills ( $f 2)$ are $\{P, V P, P\}$. While the evaluations of the third attribute with respect to the emotional steadiness are $\{P, V P, P\}$, and for the oral communication skills are $\{M P, M P, P\}$. Both are given equal weights $\{\mathrm{MH}, \mathrm{H}, \mathrm{MH}\}$. This is obvious in the with respect to the selected attributes. Resolving the same example with the absolute ideal solutions $\left\{\tilde{v}_{1}^{+}=\tilde{v}_{2}^{+}=(1,1,1,1 ; 1,1)\right.$ and $\left.\tilde{v}_{1}^{-}=\tilde{v}_{2}^{-}=(0,0,0,0 ; 1,1)\right\}$, the distances from the ideal solutions are the same for the second and third alternatives and they are equally preferred. Subsequently, it can be concluded that the relative ideal solutions are more discriminating than the absolute ideal solutions in IT2F-TOPSIS.

\section{Conclusion}

In this article, an IT2F-TOPSIS was proposed using the extended vertex method for distance measure. While the existing TOPSIS techniques for IT2FSs depend on the defuzzification of the average decision matrix or the average weighted decision matrix in the early steps, the proposed method maintains fuzziness in the preference technique to avoid any information distortion which might lead to incorrect results. First, the vertex method is extended to include IT2FSs. This distance measure is a simple formula that requires few computations. Meanwhile, the other distance measures are either inappropriate or requires extensive computations. The performance of the extended vertex method was examined using the 
32 words for computing with words proposed by Wu and Mendel [29]. The results indicate that the method is efficient in measuring the distance between IT2FSs. In addition, it has the least processing time compared to other distance measures for trapezoidal IT2FSs. Second, the fuzzy positive and negative ideal solutions are defined. Then, the relative degree of closeness to the ideal solutions is computed for each alternative. As the relative degree of closeness of an alternative increases, its preference increases. Two illustrative examples were solved using the proposed IT2F-TOPSIS. Regarding the first example, the ranking coincide with the ranking of the IVF-TOPSIS proposed by Rashid et al. [23]. As for the second example, the ranking coincides with the ranking of the IT2F-TOPSIS proposed by Chen and Lee [5]. On the other hand, when compared to the ranking of Chen and Hong [3], the second alternative was not equivalent to the third one, it proved to be better. This can be attributed to maintaining fuzziness throughout the solution steps. It was also found that the defined ideal solutions have an impact on the ranking. The relative ideal solutions are more discriminating than the absolute ideal solutions in IT2F-TOPSIS.

\section{Compliance with ethical standards}

Conflict of interest The author declares that there is no conflict of interest.

\section{References}

1. Ashtiani B, Haghighirad F, Makui A, Montazer GA (2009) Extension of fuzzy TOPSIS method based on interval-valued fuzzy sets. Appl Soft Comput 9:457-461

2. Chen C-T (2000) Extensions of the TOPSIS for group decisionmaking under fuzzy environment. Fuzzy Sets Syst 114:1-9

3. Chen S-M, Hong J-A (2014) Fuzzy multiple attributes group decision making based on ranking interval type-2 fuzzy sets and the TOPSIS method. IEEE Trans Syst Man Cybern Syst 44(12):1665-1673

4. Chen S-M, Kuo L-W (2017) Autocratic decision making using group recommendations based on interval type-2 fuzzy sets, enhanced Karnik-Mendel algorithms, and the ordered weighted aggregation operator. Inf Sci 412-413:174-193

5. Chen S-M, Lee L-W (2010) Fuzzy multiple attributes groupdecision making based on the interval type-2 TOPSIS method. Expert Syst Appl 37:2790-2798

6. Chu T-C, Lin Y-C (2003) A fuzzy TOPSIS method for robot selection. Int J Adv Manuf Technol 21:284-290

7. Das S, Kar S, Pal T (2016) Robust decision making using intuitionistic fuzzy numbers. Granul Comput 2(1):41-54

8. Dymova L, Sevastjanov P, Tikhonenko A (2015) An interval type-2 fuzzy extension of the TOPSIS methods using alpha cuts. Knowl-Based Syst 83:116-127
9. Figueroa-Garcia JC, Chalco-Cano YC, Roman-Florez H (2015) Distance measures for interval-type-2 fuzzy numbers. Discrete Appl Math 197:93-102

10. Figueroa-Garcia JC, Hernandez-Perez G (2014) On the computation of the distance between interval type-2 fuzzy numbers using a-cuts. In: IEEE conference on Norbert Wiener in the 21 st century

11. Garg H (2016) A new generalized improved score function of interval-valued intuitionistic fuzzy sets and application in expert systems. Appl Soft Comput 38:988-999

12. Ghorabaee MK (2016) Developing an MCDM method for robot selection with interval type-2 fuzzy sets. Robot Comput Integr Manuf 37:221-232

13. Heidarzade A, Mahdavi I, Mahdavi-Amiri N (2016) Supplier selection using a clustering method based on a new distance for interval type-2 fuzzy sets: a case study. Appl Soft Comput 38:213-231

14. Hwang CL, Yoon K (1981) Multiple attributes decision making methods and applications. Springer, Berlin Heidelberg

15. Ilieva G (2016) TOPSIS modification with interval type-2 fuzzy numbers. Cybern Inf Technol 16(2):60-68

16. Jiang Y, Xu Z, Shu Y (2017) Interval-valued intuitionistic multiplicative aggregation in group decision making. Granul Comput 2:387-407

17. Kahraman C, Öztayşi B, Sari IU, Turanoglu E (2014) Fuzzy analytic process with interval type-2 fuzzy sets. Knowl-Based Syst 59:48-57

18. Kumar K, Garg H (2018) TOPSIS method based on the connection number of set pair analysis under interval-valued intuitionistic fuzzy set environment. Comput Appl Math 37:1319-1329

19. Liang $Q$, Mendel J (2000) Interval-type 2 fuzzy logic systems: theory and design. IEEE Trans Fuzzy Syst 8(5):535-550

20. McCulloch J, Wagner C, Aickelin U (2013) Extending similarity measures of interval type-2 fuzzy sets to general type-2 fuzzy sets. In: IEEE international conference on fuzzy systems, Hyderabad, India

21. Niewiadomski A (2007) Interval-valued and interval type-2 fuzzy sets: a subjective comparison. IEEE, London

22. Qin J (2017) Interval type-2 fuzzy Hamy mean operators and their application in multiple criteria decision making. Granul Comput 2:249-269

23. Rashid T, Beg I, Husnine SM (2014) Robot selection by using generalized interval-valued fuzzy numbers with TOPSIS. Appl Soft Comput 21:462-468

24. Sambuc R (1975) Function $\Phi$-flous, application a l'aide au diagnostic en pathologie thyroidienne. Thèse de Doctorate en Medicine, Séction Medecine University of Marseille, Marseille, France

25. Sharaf IM (2018) TOPSIS with similarity measure for MADM applied to network selection. Comput Appl Math 37(4):4104-4121

26. Singh P (2014) Some new distance measures for type-2 fuzzy sets and distance measure based ranking for group decision making problems. Front Comput Sci 8(5):741-752

27. Singh S, Garg H (2017) Distance measures between type-2 intuitionistic fuzzy sets and their applications to multi-criteria decision-making process. Appl Intell 46:788-799

28. Sola HB, Fernandez J, Hagras $\mathrm{H}$, Herrera F, Pagola $M$, Barrenechea E (2015) Interval type 2fuzzy sets: toward a wider view on their relationship. IEEE Trans Fuzzy Syst 23(5):1876-1882

29. Wu D, Mendel JM (2009) A comparative study of ranking methods, similarity measures and uncertainty measures for interval type-2 fuzzy sets. Inf Sci 179:1169-1192 
30. Xu Z, Gou X (2017) An overview of interval-valued intuitionistic fuzzy information aggregations and applications. Granul Comput 2:13-39

31. Zadeh LH (1975) The concept of a linguistic variable and its applications to approximate reasoning. Inf Sci 8:199-249

32. Zhang $\mathrm{H}$, Zhang W, Mei C (2009) Entropy of interval-valued fuzzy sets based on distance and its relationship with similarity measure. Knowl-Based Syst 22:449-454
Publisher's Note Springer Nature remains neutral with regard to jurisdictional claims in published maps and institutional affiliations. 